\title{
Safety analysis of endoscopic haemostasis using a high-frequency live tissue electric welding device - EKVZ300 PATONMED
}

\author{
Yegor Tryliskyy ${ }^{1,2}$, Andrii Kebkalo ${ }^{1}$, Chee S. Wong ${ }^{2}$, Sergii Rublenko ${ }^{3}$, Mykhailo Rublenko³, Mykola Ilnytskiy³, \\ levgenii Negria ${ }^{4}$, Vladislav Hordovskiy ${ }^{1}$ \\ ${ }^{1}$ Department of Surgery and Proctology, Shupyk National Medical Academy of Postgraduate Education, Kyiv, Ukraine \\ 2Department of Clinical Surgery, The University of Edinburgh, Edinburgh, UK \\ ${ }^{3}$ Department of Veterinary Medicine, Bila Tserkva National Agrarian University, Bila Tserkva, Ukraine \\ ${ }^{4}$ Department of Endoscopy, Kiev City Clinical Hospital No. 8, Kyiv, Ukraine
}

Gastroenterology Rev 2018; 13 (3): 234-237

DOI: https://doi.org/10.5114/pg.2018.78289

Key words: endoscopic haemostasis, safety analysis.

Address for correspondence: Yegor Tryliskyy MSc, Department of Surgery and Proctology, Shupyk National Medical Academy of Postgraduate Education, 9 Dorohozhytska St, 03035 Kiev, Ukraine, phone: +38 0509507889, e-mail: yegor_triliskiy@ukr.net

\begin{abstract}
Introduction: The method of a bipolar high-frequency welding (HFEW) of soft living tissues of animals and humans has been used in various areas of surgery. However, it has not been utilized in endoscopic gastrointestinal procedures yet. HFEW has strong potential to be used in gastrointestinal endoscopic procedures due to the competitive cost of generator devices and due to its proven ability to coagulate vessels of wide diameter as compared to standard electrocautery devices.

Aim: To investigate the effectiveness of the endoscopic haemostasis using HFEW generator device - 300 PATONMED - in a porcine model of arterial gastrointestinal bleeding.

Material and methods: A porcine model of arterial gastrointestinal bleeding was created. A 300 PATONMED set to the "welding" regime and a flexible $7 \mathrm{Fr}$ bipolar coagulation probe with two electrodes on the tip fashioned spirally attached to convey energy were tested. Once bleeding from the artery had been initiated, the bipolar probe was applied to coagulate the bleeding site. Animals were observed for clinical evidence of recurrent bleeding and subsequently were euthanised for histological examination.

Results: A total of 10 experiments were successfully completed. An optimal haemostatic effect was achieved with durations of cautery of five to eight seconds in all animals. Continuous observation after haemostasis revealed no evidence of re-bleeding. No systemic side-effects of the technique were observed. Histological examination has shown that the peripheral thermal injury area that surrounded the coagulation zone did not spread beyond the mucosal layer in depth and $2 \mathrm{~mm}$ in width.

Conclusions: This animal study provided evidence for the safety of an HFEW in the treatment of gastrointestinal bleeding. The advantages of this technology are smokeless operative area, no tissue overheating, minimal necrosis and damage to surrounding gastric tissue, and the fact that the area of HFEW is confined to the area of the electrodes.
\end{abstract}

\section{Introduction}

Acute non-variceal upper gastrointestinal bleeding (NVUGIB) remains a common, potentially life-threatening condition. Endoscopic haemostatic therapy has been shown to improve outcomes of NVUGIB [1-3]. There are many safe and effective devices available for endoscopic haemostatic therapy in NVUGIB; for example, contact thermal devices, noncontact thermal devices, injection needles, and mechanical devices. A combination of any of the treatment modalities is commonly used to control NVUGIB. However, there are few compelling data favouring a particular device for treatment of NVUGIB, and selection of the optimal haemostatic device depends on the characteristics of the lesion, local expertise, equipment availability, and cost. The best combination for endoscopic haemostasis should obtain the highest rate of haemostasis while minimising the rates of re-bleeding and adverse events, it should be easy to use in all locations, and it should be inexpensive [4].

The method of a bipolar high-frequency welding (HFEW) of soft living tissues of animals and humans 
has been used in various areas of surgery [5-9]. The method is based on measurement of tissue impedance to accurately control the degree of coagulation and, unlike conventional bipolar coagulation technique, allows avoidance of over-heating and burning of the exposure area, sticking of the electrodes to the tissue, and the production of smoke $[10,11]$. However, HFEW has not been utilised in endoscopic gastrointestinal procedures yet. High-frequency welding has strong potential to be used in gastrointestinal endoscopic procedures due to the competitive cost of generator devices and its proven ability to coagulate vessels of wide diameter as compared to standard electrocautery devices.

\section{Aim}

The aim of this study was to investigate the safety and effectiveness of endoscopic haemostasis using a HFEW generator device - a 300 PATONMED (E.O. Paton Electric Welding Institute of the National Academy of Sciences of Ukraine, Kiev, Ukraine) - in a porcine model of massive arterial gastrointestinal bleeding.

\section{Material and methods}

\section{Preparation and development of porcine bleeding gastric ulcer model}

A porcine model of arterial gastrointestinal bleeding was created using standard technique with minor modification [12, 13]. Six healthy female domestic farm pigs (Sus scrofus domesticus) were observed for 1 week prior to study initiation, to ensure baseline health. Three days prior to the procedure, omeprazole was administered to all animals. One day prior to the procedure, omeprazole, famotidine, and antacids were given. All feeding was withheld for $12 \mathrm{~h}$ prior to the endoscopy. The baseline haematocrit, platelets, and activated clotting time (ACT) were measured in all animals prior to the procedure and were shown to be within normal range. General anaesthesia was induced with a mixture of atropine, xylazine, ketamine, and diazepam. The animals underwent tracheal intubation with mechanical ventilation, and $1.5-3.0 \%$ isoflurane in oxygen $(1.5-2.5 \mathrm{l} / \mathrm{min})$ was used to maintain anaesthesia.

All animals underwent a sterile laparotomy with freeing of a $5-\mathrm{cm}$ segment of the left gastroepiploic artery (LGA) from the place between upper and middle third of the greater curvature of the stomach wall. A 1-cm microgastrostomy was made adjacent to the free but intact blood vessel. The stomach was entered through an additional anterior 7-cm gastrostomy. Isolated LGA was tunnelled through a microgastrostomy incision with manual assistance through an anterior gastrostomic incision. The LGA was positioned and fixed so that it was exposed to the gastric lumen stretching for 3-4 cm. The gastric incisions were then closed in a standard manner along with the abdominal wall.

A GIF-140 Olympus gastroscope was inserted through the mouth of the pig and advanced into the gastric lumen to confirm the position of the artery and the air tightness of the suturing. A needle-knife was then used to incise the exposed LGA with "pure-cut" electrocautery.

Perioperatively, all animals received cefazolin (1 g) intramuscularly along with intravenous omeprazole (40 mg). Following laparotomy all animals received an intravenous bolus of $100 \mathrm{U} / \mathrm{kg}$ heparin in order to create a worst-case scenario of arterial gastrointestinal bleeding. All animals received intravenous crystalloids to maintain blood pressure above a mean $60 \mathrm{~mm} \mathrm{Hg}$. Continuous monitoring of haemodynamic parameters (heart rate, blood pressure using an arterial line) was performed.

This study was approved by the Shupyk National Medical Academy of Postgraduate Education Research Ethics Committee. It was performed between June and December 2016 at the Department of Veterinary Medicine at Bila Tserkva National Agrarian University.

\section{Endoscopic device and procedure}

An HFEW generator device - 300 PATONMED (E.O. Paton Electric Welding Institute of the National Academy of Sciences of Ukraine, Kiev, Ukraine) - set to "automatic welding" regime with electric resistance $20 \mathrm{Ohms}$ and power $200 \mathrm{~W}$ was connected to a flexible $7 \mathrm{Fr}$ bipolar coagulation probe with two electrodes on the tip fashioned spirally. The "Automatic welding" regime identifies the optimal time required to achieve complete coagulation by measuring tissue impedance using the device's software and makes a sound signal to stop coagulation. This complex was tested in the prepared animal models to coagulate arterial defects. Once bleeding from the LGA had been initiated by the means of "pure-cut" electrocautery, the bipolar probe was inserted through the channel of the gastroscope. The bipolar probe was applied perpendicularly or tangentially with mild to moderate pressure for 3 to $8 \mathrm{~s}$ to compress and coagulate the bleeding site. The bleeding spot was irrigated with normal saline to ensure completeness of haemostasis.

\section{Follow-up and post-mortem examination}

All animals were fed on the same day after the operation. They were observed for clinical evidence of recurrent bleeding. They were not put on proton pump inhibitors. All animals were euthanised 10 days after the procedure. Qualitative histologic analysis of each site of probe application was performed on formalin-fixed specimens by an independent board-certified veterinary pathologist. 


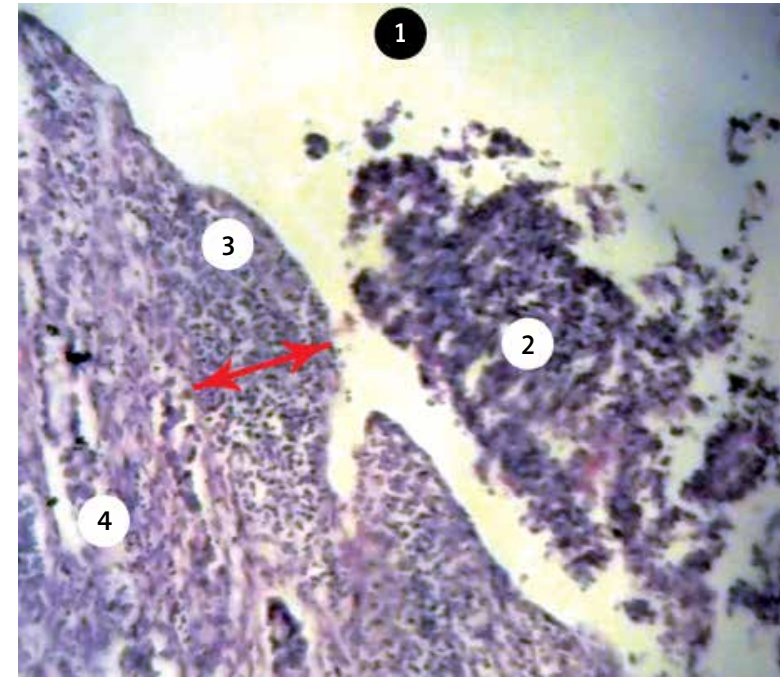

Figure 1. Multiplication 10x, H + E stain (1 gastric lumen, 2 - cellular detritus, 3 - necrotic zone, 4 - normal gastric glands)

\section{Outcomes}

The primary end point was the survival of the animals after thermal endoscopic haemostasis using HFEW technique in an ulcer bleeding model. The secondary end point included the time to achieve endoscopic haemostasis when using the HFEW-300 PATONMED device, the rate of recurrent bleeding, and the extent of local injury.

\section{Results}

A total of 10 experiments were successfully completed. Creation of an active arterial spurting haemorrhage model followed by endoscopic needle-knife injury was successful in all 10 animals. An optimal haemostatic effect was achieved with $200 \mathrm{~W}$ of power and durations of cautery of 3 and $8 \mathrm{~s}$. The process was smokeless and there was no sticking of the electrode to the coagulation zone. There was no overheating observed. Continuous observation after haemostasis revealed no evidence of re-bleeding. Repeat endoscopy on day 8 revealed no evidence of active bleeding and a healed but identifiable gastrotomy site in all animals. There was no adverse event in the survival period. All animals tolerated a full diet. Histopathological findings showed a precise demarcated coagulation zone at the LGA wall, which matched the size of the electrode. This zone was surrounded by peripheral tissue injury. This injury was manifested by destruction of epithelial cells of the outer zone of gastric glands and leukocytic mucosal infiltration reaching a maximum depth of $0.5 \mathrm{~mm}$. Deep parts of glands were mostly not involved, and in all cases the maximum spread of injury laterally was $2 \mathrm{~mm}$ (Figure 1).

\section{Discussion}

This animal study provides evidence for the safety of a HFEW in the treatment of active, severe, NVUGIB. The size of an artery is one of the main determinants of the success of endoscopic haemostasis. Our model simulated bleeding ulcers with a large vessel at the base. Therefore, an ulcer model with haemorrhage commonly refractory to existing endoscopic devices was created. Initial haemostasis was achieved in all animals, and there were no systemic or local procedure-related complications. The procedure was smokeless, and there was no sticking of the electrode to the tissue - this was due to the device's ability to control coagulation and to avoid overheating. In all cases the depth of the injury did not spread beyond $0.5 \mathrm{~mm}$, due to the nature of the controlled thermal effect of the device, and hence low risk of gastric perforation.

The artery diameter that was successfully coagulated is comparable to the size of the gastroduodenal artery in humans. However, the chronicity of ulcer characterised by hard fibrotic tissue could not be achieved in this experiment. The technique is operator dependant like all contact thermal haemostatic techniques, hence potential obstacles related to operator experience and bleeding location in achieving haemostasis may arise in clinical practice. The learning curve of this technique was not specifically assessed in this study, but it may be comparable to the learning curve of all endoscopic contact thermal haemostatic devices. Cost effectiveness analysis of this technique in comparison to conventional techniques has not been performed, but based on the market price of the generator device at the time of publication, the 300 PATONMED has a more favourable price when comparing it to other known bipolar and argon-plasma cautery devices.

\section{Conclusions}

From the results of the study, we believe that the HFEW generator device, the 300 PATONMED, provides a feasible and reliable technique in achieving endoscopic haemostasis on bleeding peptic ulcers. There are several advantages of this technique over conventional methods. However, the data provide only limited conclusions. The next logical step would be to assess clinical application of the 300 PATONMED in humans.

\section{Conflict of interest}

The authors declare no conflict of interest.

\section{References}

1. Cook DJ, Guyatt GH, Salena BJ, Laine LA. Endoscopic therapy for acute nonvariceal upper gastrointestinal hemorrhage: a meta-analysis. Gastroenterology 1992; 102: 139-48. 
2. Sacks HS, Chalmers TC, Blum AL, et al. Endoscopic hemostasis. An effective therapy for bleeding peptic ulcers. JAMA 1990; 264: 494-9.

3. Sung JJ, Tsoi KK, Lai LH, et al. Endoscopic clipping versus injection and thermo-coagulation in the treatment of non-variceal upper gastrointestinal bleeding: a meta-analysis. Gut 2007; 56: 1364-73.

4. Aslanian HR, Laine L. Hemostatic powder spray for Gl bleeding. Gastrointest Endosc 2013; 77: 508-10.

5. Han S, Cai Z, Ning X, et al. Comparison of a new high-frequency electric welding system for intestinal closure with handsewn in vivo pig model. J Laparoendosc Adv Surg Techn A 2015; 25: 662-7.

6. Linchevskyy O, Makarov A, Getman V. Lung sealing using the tissue-welding technology in spontaneous pneumothorax. Eur J Cardiothorac Surg 2010; 37: 1126-8.

7. Umanets N, Pasyechnikova NV, Naumenko VA, Henrich PB. High-frequency electric welding: a novel method for improved immediate chorioretinal adhesion in vitreoretinal surgery. Graefes Arch Clin Exp Ophthalmol 2014; 252: 1697-703.

8. Patton B, Lebedev V, Furmanov Y, et al. Instrument and method for the end-to-end reconnection of intestinal tissues. Google Patents 2007.

9. Paton BE, Lebedev VK, Furmanov YA, et al. Instrument and method for reconnection of intestinal tissues. Google Patents 2008.

10. Paton BE, Lebedev VK, Lebedev AV, et al. System and method for control of tissue welding. Google Patents 2003.

11. Paton B, Lebedev V, Vorona D, et al. Bonding of soft biological tissues by passing high frequency electric current therethrough. Google Patents 2005.

12. Giday SA, Kim Y, Krishnamurty DM, et al. Long-term randomized controlled trial of a novel nanopowder hemostatic agent (TC-325) for control of severe arterial upper gastrointestinal bleeding in a porcine model. Endoscopy 2011; 43: 296-9.

13. Kebkalo A, Rublenko M, Rublenko S, et al. Experimental model of massive gastrointestinal bleeding: methodology aspects. Clin Exp Pathol 2016; 57: 47-54.

Received: 26.09 .2017

Accepted: 7.06 .2018 